\title{
A Proof of the Riemann Hypothesis Based on Hadamard Product
}

\author{
Prof. Fayez A. Alhargan* \\ Revised August 6, 2020
}

Keywords:the Riemann Hypothesis, the functional equation, the Riemann zeta function, Hadamard Product.

2000 Mathematics Subject Classification:11M26

\begin{abstract}
By unraveling a persistent misconception in the zeta Hadamard product expansion, and employing the zeta functional equation, a concise proof of the Riemann Hypothesis is presented, which conclusively demonstrate that the Riemann Hypothesis is true.
\end{abstract}

\section{Introduction}

The Riemann hypothesis is a conjecture that the Riemann zeta function $\zeta(s)$ has its zeros only at the negative even integers and complex numbers with real part $\frac{1}{2}$. It was proposed by Bernhard Riemann (1859)[1]. Jacques Hadamard in (1893) [2], based on Weierstrass's factorization theorem, showed that the Riemann zeta function $\zeta(s)$, can be expressed as an infinite product expansion over the non-trivial zeros of the zeta function. The Riemann Hypothesis is the eighth problem in David Hilbert's list of 23 unsolved problems published in (1900) [3], there has been tremendous work on the subject since then, which are illustrated in Titchmarsh (1930) [4], Edwards (1975) [5], Ivic (1985) [6] and Karatsuba (1992)[7]. However, it is still regarded as one of the most difficult unsolved problems and has been placed as the second most important problem in the list of the Clay Mathematics Institute Millennium Prize Problems (2000), as its proof would shed light on many of the mysteries surrounding the distribution of prime numbers [8] and [9].

The Riemann zeta function is the function of the complex variable $s$, defined in the half-plane $\Re(s)>1$ by the absolutely convergent series

$$
\zeta(s):=\sum_{n=1}^{\infty} \frac{1}{n^{s}}
$$

*PhD, BEng. Senior Researcher at PSDSARC, email:fayez.alhargan@psdsarc.org.sa. 
and in the whole complex plane by analytic continuation [8].

The Riemann hypothesis is concerned with the locations of the non-trivial zeros of $\zeta(s)$, and states that: The nontrivial zeros of $\zeta(s)$ have real part equal to $\frac{1}{2}[8]$.

In this paper, I will prove the truth of the Riemann Hypothesis by employing the Hadamard product and the functional equation of the zeta function. The proof is given in a less abstract form and includes some 'simplistic steps' for it to be accessible to a wider audience.

\section{Proof of the Riemann Hypothesis}

Theorem 1 The Riemann zeta function $\zeta(s)$ has only two independent sets of principle zeros $M$ and $S$. The set $M$ of all principle trivial zeros of $\zeta(s)$ is on the real negative axis with the imaginary part $t=0$, whereas the set $S$ of all principle non-trivial zeros of $\zeta(s)$ is on the imaginary line with the real part $\sigma=\frac{1}{2}$.

\section{Proof:}

It was shown by Riemann [1], that the zeta function satisfies the following functional equation

$$
\zeta(s)=2^{s} \pi^{s-1} \sin \left(\frac{\pi s}{2}\right) \Gamma(1-s) \zeta(1-s),
$$

in addition, we have the well known fact that

$$
\zeta(s)=\overline{\zeta(\bar{s})}
$$

where $s=\sigma+i t$ is a complex variable with $\sigma$ and $t$ being real numbers, and $i$ is the imaginary unit.

Now, if $\zeta(s)=0$, then equations (2) and (3) must be zero, thus

$$
\begin{aligned}
& \zeta(s)=2^{s} \pi^{s-1} \sin \left(\frac{\pi s}{2}\right) \Gamma(1-s) \zeta(1-s)=0, \\
& \zeta(s)=\overline{\zeta(\bar{s})}=0 .
\end{aligned}
$$

Therefore, from equation (5) all the zeros of the zeta function are conjugates, and from equation (4) the zeros must satisfy

$$
\sin \left(\frac{\pi s}{2}\right) \Gamma(1-s)=0,
$$

or

$$
\zeta(s)=0 .
$$

From equation (6), we obtain the set $M$ of all the principle trivial zeros of $\zeta(s)$, i.e. $M=\{-2,-4, \ldots,-2 m, \ldots\}$, where $m$ is a positive integer. And from equation (7), we obtain another independent set $S$ of all the principle non-trivial 
zeros of $\zeta(s), S=\left\{s_{1}, s_{2}, \ldots, s_{r}, \ldots\right\}$, with the $r$ th zero $s_{r}=\sigma_{r}+i t_{r}$, where $0<\sigma_{r}<1$, and $t_{r}$ are real numbers, and $i$ is the imaginary unit.

Furthemore, we note from equations (4) and (5), that the set $S$ of all the principle non-trivial zeros $S$ and the set $M$ of all the principle trivial zeros of $\zeta(s)$, these two sets $S$ and $M$, must satisfy as well both $\zeta(1-s)=0$ and $\overline{\zeta(\bar{s})}=0$, i.e. $\zeta\left(1-s_{r}\right)=0, \overline{\zeta\left(\bar{s}_{r}\right)}=0, \zeta(1+2 m)=0$ and $\overline{\zeta(\overline{-2 m})}=0$.

Now using the Legendre duplication formula for $\Gamma(z)$, we have

$$
\Gamma\left(\frac{1}{2}-\frac{s}{2}\right)=\frac{2^{s} \pi^{\frac{1}{2}} \Gamma(1-s)}{\Gamma\left(1-\frac{s}{2}\right)}
$$

and since

$$
\pi^{-1} \sin \left(\frac{\pi s}{2}\right) \Gamma(1-s)=\frac{\Gamma(1-s)}{\Gamma\left(1-\frac{s}{2}\right) \Gamma\left(\frac{s}{2}\right)}
$$

thus, we have

$$
\pi^{-1} \sin \left(\frac{\pi s}{2}\right) \Gamma(1-s)=\frac{\pi^{-\frac{1}{2}} \Gamma\left(\frac{1}{2}-\frac{s}{2}\right)}{2^{s} \Gamma\left(\frac{s}{2}\right)} .
$$

Therefore, the functional equation (2) becomes

$$
\frac{\zeta(s)}{\zeta(1-s)}=\pi^{s-\frac{1}{2}} \frac{\Gamma\left(\frac{1}{2}-\frac{s}{2}\right)}{\Gamma\left(\frac{s}{2}\right)}
$$

Now, consider the Hadamard product [2], which is an infinite product expansion over the principle non-trivial zeros $s_{r}$ of the zeta function, given by

$$
\zeta(s)=\pi^{\frac{s}{2}} \frac{\prod_{r}\left(\frac{s_{r}-s}{s_{r}}\right)\left(\frac{\bar{s}_{r}-s}{\bar{s}_{r}}\right)}{(s-1) s \Gamma\left(\frac{s}{2}\right)},
$$

also we can obtain the Hadamard product expansion over both the set of the trivial zeros $M$ and the set of the non-trivial zeros $S$, by noting that

$$
\frac{1}{\Gamma\left(\frac{s}{2}\right)}=\frac{s}{2} e^{\gamma \frac{s}{2}} \prod_{m=1}^{\infty}\left(1+\frac{s}{2 m}\right) e^{-\frac{s}{2 m}}
$$

where $\gamma$ is the Euler-Mascheroni constant.

Thus, we have

$$
\zeta(s)=\frac{\pi^{\frac{s}{2}} e^{\gamma \frac{s}{2}}}{2(s-1)} \prod_{r=1}^{\infty}\left(1-\frac{s}{s_{r}}\right)\left(1-\frac{s}{\bar{s}_{r}}\right)\left(1+\frac{s}{2 r}\right) e^{-\frac{s}{2 r}} .
$$

It is worh noting that there is some misconception in the literature about the zeros that should be included in the product expansions in eqaution (11). 
Many assume that the product over the zeros includes the non-principle zeros of $\zeta(s)$, i.e. $s=1-s_{r}$ and $s=1-\bar{s}_{r}$, in the Hadamard product expansion (11). Such assunption is incorrect, for in this case we should also include the nonprinciple trivial zeros $s=2+2 m$ in the product expansion (12) and $s=1+2 m$ in the product expansion (13), as $s=2+2 m$ are also zeros of $1 / \Gamma\left(1-\frac{s}{2}\right)$, and $s=1+2 m$ are also zeros of $\zeta(1-s)$. Clearing this misconception is key to the understanding of the proof of the theorem.

Now, from equation (11), we have

$$
\zeta(s)=\pi^{\frac{s}{2}} \frac{\prod_{r}\left(\frac{s_{r}-s}{s_{r}}\right)\left(\frac{\bar{s}_{r}-s}{\bar{s}_{r}}\right)}{(s-1) s \Gamma\left(\frac{s}{2}\right)},
$$

and since $s=1-s_{r}$ are principle zeros of $\zeta(1-s)$, then we have the Hadamard product expansion for $\zeta(1-s)$ as follows

$$
\zeta(1-s)=\pi^{\frac{1-s}{2}} \frac{\prod_{r}\left(\frac{s_{r}-s}{s_{r}-1}\right)\left(\frac{\bar{s}_{r}-s}{\bar{s}_{r}-1}\right)}{s(1-s) \Gamma\left(\frac{1}{2}-\frac{s}{2}\right)} .
$$

Note that in equation (15), the zeros $s=s_{r}$ are not including in the product expansion of $\zeta(1-s)$, as the zeros $s=s_{r}$ are not principle zeros of $\zeta(1-s)$.

Now, dividing equation (14) by equation (15), we have

$$
\frac{\zeta(s)}{\zeta(1-s)}=\pi^{s-\frac{1}{2}} \frac{\Gamma\left(\frac{1}{2}-\frac{s}{2}\right)}{\Gamma\left(\frac{s}{2}\right)} \frac{\prod_{r}\left(\frac{s_{r}-s}{s_{r}}\right)\left(\frac{\bar{s}_{r}-s}{\bar{s}_{r}}\right)}{\prod_{r}\left(\frac{s_{r}-s}{s_{r}-1}\right)\left(\frac{\bar{s}_{r}-s}{\bar{s}_{r}-1}\right)}
$$

i.e.

$$
\frac{\zeta(s)}{\zeta(1-s)}=\pi^{s-\frac{1}{2}} \frac{\Gamma\left(\frac{1}{2}-\frac{s}{2}\right)}{\Gamma\left(\frac{s}{2}\right)} \prod_{r}\left(\frac{s_{r}-1}{s_{r}}\right)\left(\frac{\bar{s}_{r}-1}{\bar{s}_{r}}\right),
$$

we note that

$$
\left(\frac{s_{r}-1}{s_{r}}\right)\left(\frac{\bar{s}_{r}-1}{\bar{s}_{r}}\right)=\left(1+\frac{1-2 \sigma_{r}}{s_{r} \bar{s}_{r}}\right),
$$

which gives us

$$
\frac{\zeta(s)}{\zeta(1-s)}=\pi^{s-\frac{1}{2}} \frac{\Gamma\left(\frac{1}{2}-\frac{s}{2}\right)}{\Gamma\left(\frac{s}{2}\right)} \prod_{r}\left(1+\frac{1-2 \sigma_{r}}{s_{r} \bar{s}_{r}}\right) .
$$

Now, equating (10) and (19), we have

$$
\frac{\pi^{s-\frac{1}{2}} \Gamma\left(\frac{1}{2}-\frac{s}{2}\right)}{\Gamma\left(\frac{s}{2}\right)} \prod_{r}\left(1+\frac{1-2 \sigma_{r}}{s_{r} \bar{s}_{r}}\right)=\frac{\pi^{s-\frac{1}{2}} \Gamma\left(\frac{1}{2}-\frac{s}{2}\right)}{\Gamma\left(\frac{s}{2}\right)},
$$


which simplifies to

$$
\prod_{r=1}^{\infty}\left(1+\frac{1-2 \sigma_{r}}{s_{r} \bar{s}_{r}}\right)=1 .
$$

Now, equation (21) is true only if $1-2 \sigma_{r}=0$, which gives $\sigma_{r}=\frac{1}{2}$, for all the non-trivial zeros of $\zeta(s)$, concluding the proof of the Riemann Hypothesis, that the real part of every non-trivial zero of the Riemann zeta function is $\sigma_{r}=\frac{1}{2}$.

\section{Conclusion}

The proof of the Riemann Hypothesis would unravel many of the mysteries surrounding the distribution of prime numbers, as the primes are at the heart of all encryption systems. Furthermore, the proof of the Riemann Hypothesis would as a consequence, prove many of the propositions known to be true under the Riemann Hypothesis. The proof shown in this paper was based on a basic insight into the relation of the Hadamard product expansion and the functional equation of the Riemann zeta function, which were available from Hadamard's publication in (1893) and Riemann's first publication in (1859). Sometimes the truth is hidden in plain sight.

\section{References}

[1] Riemann, Bernhard (1859). "Über die Anzahl der Primzahlen unter einer gegebenen Grösse”. Monatsberichte der Berliner Akademie.. In Gesammelte Werke, Teubner, Leipzig (1892), Reprinted by Dover, New York (1953).

[2] J. Hadamard (1893). "Etude sur les propriétés des fonctions entières et en particulier d'une fonction considérée par Riemann". Journal de Mathématiques Pures et Appliquées.

[3] Hilbert, David (1900), Mathematische Probleme, Gottinger Nachrichten, pp. 253-297.

[4] Titchmarsh, E. C. (1986). Heath-Brown (ed.) The Theory of the Riemann Zeta Function (2nd rev. ed.), Oxford University Press.

[5] Edwards, H. M. (1974). Riemann's Zeta Function, Academic Press.

[6] Ivic, A. (1985). The Riemann Zeta Function, John Wiley \& Sons.

[7] Karatsuba, A. A.; Voronin, S. M. (1992). The Riemann Zeta-Function Berlin: W. de Gruyter.

[8] Bombieri, Enrico (2000). "Problems of the millennium: The Riemann Hypothesis", CLAY.

[9] Sarnak, Peter (2004). "Problems of the Millennium: The Riemann Hypothesis", CLAY. 